\title{
Multidimensional data in multidimensional scaling using the analytic network process
}

\author{
Jih-Jeng Huang a , Gwo-Hshiung Tzeng ${ }^{\text {b,c,* }}$, Chorng-Shyong Ong ${ }^{\text {a }}$ \\ a Department of Information Management, National Taiwan University, No. 1, Sec. 4, Roosevelt Road, Taipei 106, Taiwan \\ ${ }^{\mathrm{b}}$ Institute of Management of Technology and Institute of Traffic and Transportation College of Management, \\ National Chiao Tung University, 1001 Ta-Hsueh Road, Hsinchu 300, Taiwan \\ ${ }^{\mathrm{c}}$ Kainan University, No. 1, Kainan Road, Luchu, Taoyuan 338, Taiwan
}

Received 24 December 2003; received in revised form 24 August 2004

Available online 2 November 2004

\begin{abstract}
Multidimensional scaling (MDS) is a statistical tool for constructing a low-dimensional configuration to represent the relationships between the objects. Although MDS has been widely used in various fields, it is difficult to evaluate similarity/ dissimilarity between the complex systems by human judgment. Even though we can divide a complex system into subsystems, which can be more easily evaluated, the relative weights of the subsystems are also a crucial problem. Because of these subsystems usually exist interdependence and feedback, the weights of the subsystems are hard to obtain. This paper proposes a method which combines the methods of the interpretive structural modeling (ISM) and the analytic network process (ANP) procedures to deal with the problem of the subsystems' interdependence and feedback. In addition, we also provide a numerical example to illustrate the proposed method.
\end{abstract}

(c) 2004 Elsevier B.V. All rights reserved.

Keywords: Multidimensional scaling (MDS); Interdependence; Feedback; Interpretive structural modeling (ISM); Analytic network process (ANP)

\footnotetext{
* Corresponding author. Address: Institute of Management of Technology and Institute of Traffic and Transportation College of Management, National Chiao Tung University, 1001 Ta-Hsueh Road, Hsinchu 300, Taiwan. Tel.: +886 3 571212157505; fax: +88635753926.

E-mail address: ghtzeng@cc.nctu.edu.tw (G.-H. Tzeng).
}

\section{Introduction}

Multidimensional scaling (MDS) is used to represent the relationships between the objects by constructing a configuration of $n$ points in lowdimension from the pairwise comparisons of the dissimilarities among a set of $n$ objects (Arabic et al., 1987; Young and Hamer, 1987; Schiffman et al., 1981). The dissimilarities can be calculated 
by Euclidean distance or other weighted Euclidean distance (Kruskal and Wish, 1978) to form the proximity matrix. General speaking, MDS can be classified into metric MDS and non-metric MDS based on the type of the input data or whether or not the distance matrix satisfies Euclidean distance. The goal of metric MDS and non-metric MDS is to visually reflect the empirical relationships in the data. More detailed discussions about MDS can refer to Mead (1992).

Although MDS has been widely used in various fields, such as psychophysics, sensor analysis or marketing, the problems of the feature's weights have received little attention in social science. In conventional MDS, the proximity matrix is given subjectively by asking such question as "How many grades of dissimilarity do you think there are between object 1 and object 2?" However it is difficult for human to evaluate the dissimilarities in the complex systems. Even though we can divide a complex system into many subsystems which can be easily evaluated, the weights of the subsystems also is a hard problem because of existing interdependence and feedback relationships.

This paper proposes a method which combines the methods of the interpretive structural modeling (ISM) and the analytic network process (ANP) procedures to deal with the problem of the subsystems' interdependence and feedback. We also illustrate a numerical example to show the steps of the proposed method. On the basis of the results, we can conclude that the weights play a key for the MDS analysis. In addition, the proposed method can provide the more informative and accurate results than the conventional MDS analysis.

The rest of this paper is organized as follows. Section 2 states the problems in judging the proximity matrix. Section 3 describes the ANP method and ISM is presented in Section 4. A numerical example is illustrated in Section 5. Discussions and conclusions are presented in Section 6 and Section 7 , respectively.

\section{Problem descriptions}

The problem of measurement can be described as follows. If we want to analyze the relationships between the countries (such as the USA, the UK, Japan, and so on) using MDS analysis, we can construct the proximity matrix by our objective/ subjective judgment. However the problem arises of what are the criteria used to judge these grades of dissimilarity between the countries. It is clear that with the different criteria, the results are various. However, in the conventional MDS method, we do not understand these criteria or their relationships even though the information is important for decision-makers or researchers. In practice, we usually have some information which could be used to make the results more accurate and objective in MDS analysis.

It is clear that in a complex system or construct, it is difficult for human to quantify a precise value in the proximity matrix. However, we could divide a complex system into many elements or subsystems which can be easily judged to measure the grades of elements. Then, sum these grades to obtain the final proximity matrix. The advantage of this method is that we can more easily judge the differences between the elements and understand what criteria are based on.

Although it seems rational that we can sum the grades of these elements to calculate the proximity matrix among the elements, the weights between these elements may not be the same. It is clear that these elements may have interdependent or feedback relationships. It may distort the actual results if we assume the weights of the elements are equal. In this paper, ISM and ANP are used here to overcome these problems. First, ISM is used to construct the network relationships between elements, and then the ANP is used to calculate the weights of the elements for solving above problems.

\section{The analytic network process}

The ANP was proposed in (Saaty, 1996; Saaty and Vargas, 1998) to overcome the problem of interdependence and feedback between criteria or alternatives. The ANP is the general form of the analytic hierarchy process (AHP) (Saaty, 1980) which has been used in multicriteria decision making $(\mathrm{MCDM})$ to release the restriction of hierarchical structure, and has been applied to project 
selection (Meade and Presley, 2002; Lee and Kim, 2000), product planning, strategic decision (Sarkis, 2003; Karsak et al., 2002), optimal scheduling (Momoh and Zhu, 2003) and so on.

The first phase of the ANP is to compare the criteria in whole system to form the supermatrix. This is done through pairwise comparisons by asking "How much importance does a criterion have compared to another criterion with respect to our interests or preferences?" The relative importance value can be determined using a scale of 1 to 9 to represent equal importance to extreme importance (Saaty, 1980, 1996). The general form of the supermatrix can be described as follows:

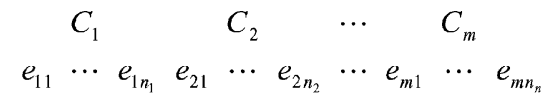

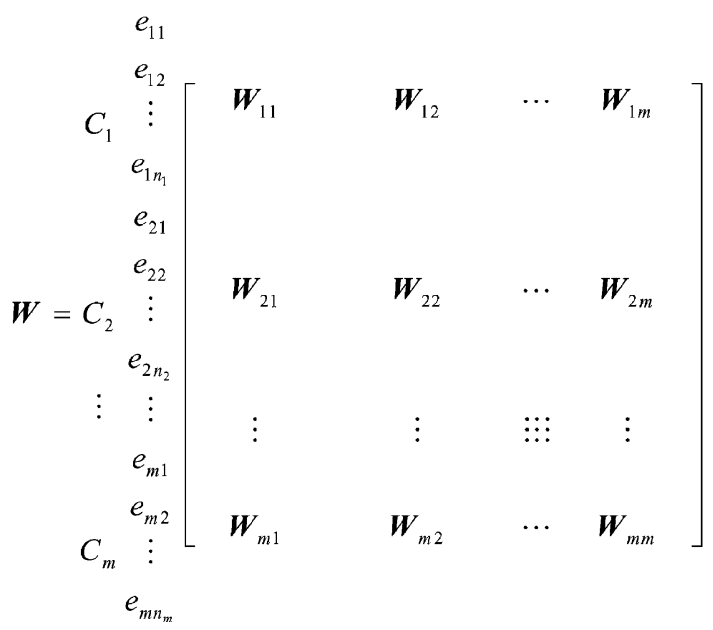

where $C_{m}$ denotes the $m$ th cluster, $e_{m n}$ denotes the $n$th element in $m$ th cluster, and $\boldsymbol{W}_{i j}$ is the principal eigenvector of the influence of the elements compared in the $j$ th cluster to the $i$ th cluster. In addition, if the $j$ th cluster has no influence to the $i$ th cluster, then $\boldsymbol{W}_{i j}=0$.

Therefore, the form of the supermatrix depends much on the variety of the structure. There are several structures which were proposed by Saaty including hierarchy, holarchy, suparchy, intarchy, etc. to demonstrate how the structure is affected by the supermatrix. Here, two simple cases, which both have three clusters, are used to show how to form the supermatrix based on the structures (Fig. 1).

The supermatrix can be formed as the following matrix:

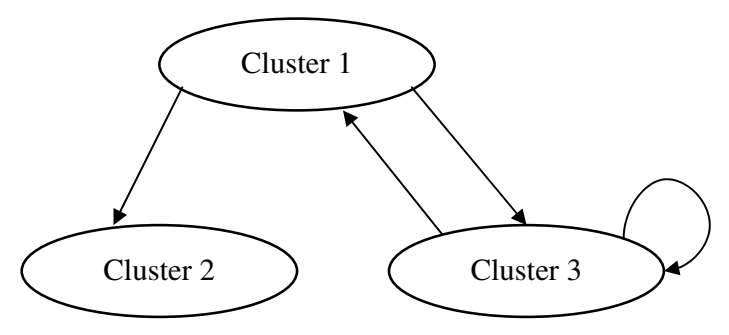

Fig. 1. The structure of the case 1 .

$$
\begin{aligned}
& C_{1} \quad C_{2} \quad C_{3} \\
& \boldsymbol{W}=\begin{array}{c}
C_{1} \\
C_{2} \\
C_{3}
\end{array}\left[\begin{array}{ccc}
0 & 0 & W_{13} \\
W_{21} & 0 & 0 \\
W_{31} & 0 & W_{33}
\end{array}\right]
\end{aligned}
$$

In Fig. 2, the second case is more complex than the first case.

Then, the supermatrix of the second case can be expressed as

$$
\begin{aligned}
& \begin{array}{lll}
C_{1} & C_{2} & C_{3}
\end{array} \\
& \boldsymbol{W}=\begin{array}{l}
C_{1} \\
C_{2} \\
C_{3}
\end{array}\left[\begin{array}{ccc}
W_{11} & W_{12} & W_{13} \\
W_{21} & W_{22} & 0 \\
0 & W_{32} & 0
\end{array}\right]
\end{aligned}
$$

After forming the supermatrix, the weighted supermatrix is derived by transforming all columns sum to unity exactly. This step is much similar to the concept of Markov chain for ensuring the sum of these probabilities of all states equals to 1. Next, we raise the weighted supermatrix to limiting powers such as Eq. (1) to get the global priority vectors or called weights.

$\lim _{k \rightarrow \infty} \boldsymbol{W}^{k}$

In addition, if the supermatrix has the effect of cyclicity, the limiting supermatrix is not the only one.

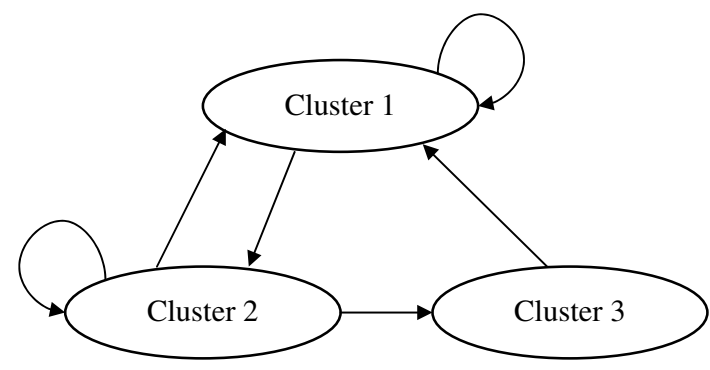

Fig. 2. The structure of the case 2 . 
There are two or more limiting supermatrices in this situation, and the Cesaro sum would be calculated to get the priority. The Cesaro sum is formulated as

$\lim _{k \rightarrow \infty}\left(\frac{1}{N}\right) \sum_{k=1}^{N} \boldsymbol{W}^{k}$

to calculate the average effect of the limiting supermatrix (i.e. the average priority weights). Otherwise, the supermatrix would be raised to large powers to get the priority weights. The detailed discussion of the mathematical processes of the ANP can refer to Saaty (1996) and Sekitani and Takahashi (2001).

In order to show the concrete procedures of the ANP, a simple example of system development is demonstrated to derive the priority of each criterion. As we know, the key to develop a successful system depending on the match of human and technology factors. Assume the human factor can be measured by the criteria of business culture $(C)$, end-user demand $(E)$ and management $(M)$. On the other hand the technology factor can be measured by the criteria of employee ability $(A)$, process $(P)$ and resource $(R)$. In addition, human and technology factors are affected each other as like as the structure shown in Fig. 3.

The first step of the ANP is to compare the importance between each criterion. For example, the first matrix below is to ask the question "For the criterion of employee ability, how much the importance does one of the human criteria than another". The other matrices can easily be formed with the same procedures. The next step is to calculate the influence (i.e. calculate the principal eigenvector) of the elements (criterion) in each component (matrix).

\begin{tabular}{llllll}
\hline Ability & Culture & End-user & Management & Eigenvector & Normalization \\
\hline Culture & 1 & 3 & 4 & 0.634 & 0.634 \\
End-user & $1 / 3$ & 1 & 1 & 0.192 & 0.192 \\
Management & $1 / 4$ & 1 & 1 & 0.174 & 0.174
\end{tabular}

Process

\begin{tabular}{llllll}
\hline Culture & 1 & 1 & $1 / 2$ & 0.250 & 0.250 \\
End-user & 1 & 1 & $1 / 2$ & 0.250 & 0.250 \\
Management & 2 & 2 & 1 & 0.500 & 0.500
\end{tabular}

Resource

\begin{tabular}{llllll}
\hline Culture & 1 & 2 & 1 & 0.400 & 0.400 \\
End-user & $1 / 2$ & 1 & $1 / 2$ & 0.200 & 0.200 \\
Management & 1 & 2 & 1 & 0.400 & 0.400
\end{tabular}

\begin{tabular}{llllll} 
Culture & Ability & Process & Resource & & \\
\cline { 1 - 3 } Ability & 1 & 5 & 3 & 0.637 & 0.637 \\
Process & $1 / 5$ & 1 & $1 / 3$ & 0.105 & 0.105 \\
Resource & $1 / 3$ & 3 & 1 & 0.258 & 0.258
\end{tabular}

End-user

\begin{tabular}{llllll}
\cline { 1 - 1 } Ability & 1 & 5 & 2 & 0.582 & 0.582 \\
Process & $1 / 5$ & 1 & $1 / 3$ & 0.109 & 0.109 \\
Resource & $1 / 2$ & 3 & 1 & 0.309 & 0.309
\end{tabular}

Management

\begin{tabular}{llllll}
\hline Ability & 1 & $1 / 5$ & $1 / 3$ & 0.136 & 0.136 \\
Process & 5 & 1 & 3 & 0.654 & 0.654 \\
Resource & 3 & $1 / 3$ & 1 & 0.210 & 0.210 \\
\hline
\end{tabular}




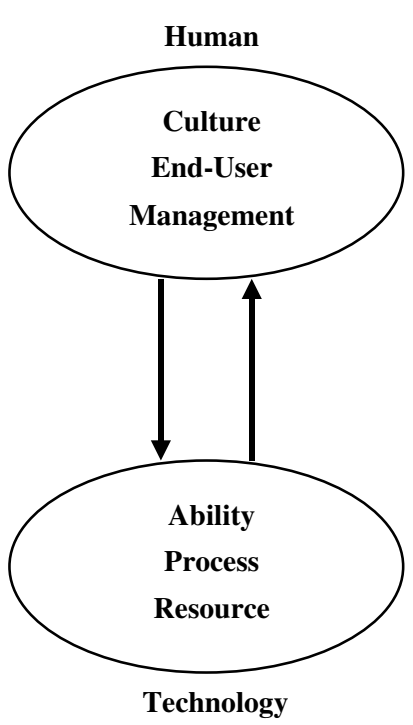

Fig. 3. The structure of the system development.

Now, we can form the supermatrix based on the above eigenvectors and the structure in Fig. 3. Since the human factor can affect the technology factor, and vise versa, the supermatrix is formed as follows:

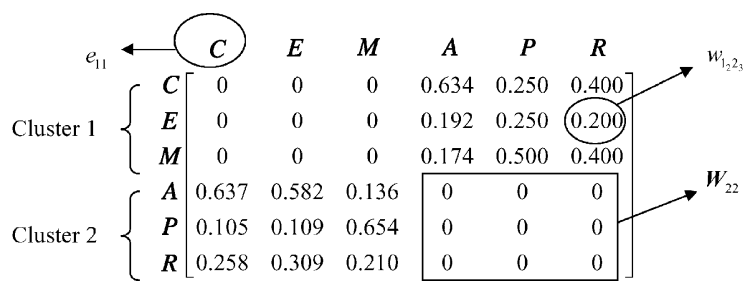

Then, the weighted supermatrix is obtained by ensuring all columns sum to unity exactly.

\begin{tabular}{|c|c|c|c|c|c|c|}
\hline & $C$ & $E$ & $M$ & $A$ & $P$ & $R$ \\
\hline$C$ & 0 & 0 & 0 & 0.634 & 0.250 & $0.400]$ \\
\hline$E$ & 0 & 0 & 0 & 0.192 & 0.250 & 0.200 \\
\hline 1 & 0 & 0 & 0 & 0.174 & 0.500 & 0.400 \\
\hline$A$ & 0.637 & 0.582 & 0.136 & 0 & 0 & 0 \\
\hline 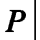 & 0.105 & 0.109 & 0.654 & 0 & 0 & 0 \\
\hline & 0.258 & 0.309 & 0.210 & 0 & 0 & 0 \\
\hline
\end{tabular}

Last, by calculating the limiting power of the weighted supermatrix, the limiting supermatrix is obtained as follows:

$\boldsymbol{C}$
$\boldsymbol{E}$
$\boldsymbol{M}$
$\boldsymbol{A}$
$\boldsymbol{P}$
$\boldsymbol{P}$
$\boldsymbol{R}$$\left[\begin{array}{cccccc}\boldsymbol{C} & \boldsymbol{M} & \boldsymbol{M} & \boldsymbol{P} & \boldsymbol{R} \\ 0 & 0 & 0 & 0.464 & 0.464 & 0.464 \\ 0 & 0 & 0 & 0.324 & 0.324 & 0.324 \\ 0.463 & 0.463 & 0.463 & 0 & 0 & 0 \\ 0.284 & 0.284 & 0.284 & 0 & 0 & 0 \\ 0.253 & 0.253 & 0.253 & 0 & 0 & 0\end{array}\right]$

(When $k$ is even)

and

\begin{tabular}{|c|c|c|c|c|c|c|}
\hline & $C$ & $E$ & $M$ & $A$ & $P$ & $R$ \\
\hline$C$ & {$[0.464$} & 0.464 & 0.464 & 0 & 0 & 0 \\
\hline$E$ & 0.210 & 0.210 & 0.210 & 0 & 0 & 0 \\
\hline$M$ & 0.324 & 0.324 & 0.324 & 0 & 0 & 0 \\
\hline 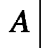 & 0 & 0 & 0 & 0.463 & 0.463 & 0.463 \\
\hline$P$ & 0 & 0 & 0 & 0.284 & 0.284 & 0.284 \\
\hline$R$ & 0 & 0 & 0 & 0.253 & 0.253 & 0.253 \\
\hline
\end{tabular}

(When $k$ is odd)

As we see, the supermatrix has the effect of cyclicity, and the Cesaro sum (i.e. add the two matrices and dividing by two) is used here to obtain the final priorities as follows:

\begin{tabular}{|c|c|c|c|c|c|c|}
\hline & $C$ & $E$ & $M$ & $A$ & $P$ & $R$ \\
\hline$C$ & 0.233 & .233 & 0.233 & 0.233 & 0.233 & 0.2 \\
\hline & 105 & 0.105 & 0.105 & 0.105 & 0.105 & 0.105 \\
\hline & 0.162 & 0.162 & 0.162 & 0.162 & 0.162 & 0.162 \\
\hline & 0.231 & 0.231 & 0.231 & 0.2 & 0.231 & 0.23 \\
\hline & 0.142 & 0.142 & 0.142 & 0.142 & 0.142 & 0.142 \\
\hline & 127 & 0.127 & 0.127 & 0.127 & 0.127 & 0.12 \\
\hline
\end{tabular}

In this example, the criterion of culture has the highest priority $(0.233)$ in system development and the criterion of end-user has the least priority (0.105).

In order to show the effect of the structure in the ANP, the other structure, which has the feedback effect on human factors, is considered as in Fig. 4.

There are two methods to deal with the selffeedback effect. The first method simply place 1 in diagonal elements and the other method performs a pairwise comparison of the criteria on each criterion. In this example, we use the first method. With the same steps above, the unweighted 


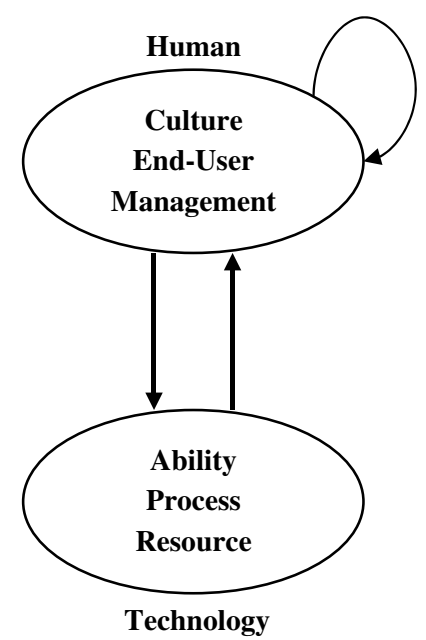

Fig. 4. The structure of system development with feedback effects.

supermatrix, the weighted supermatrix, and the limiting supermatrix can be obtained as follows, respectively:

\begin{tabular}{|c|c|c|c|c|c|c|}
\hline & $C$ & $E$ & $M$ & $A$ & $P$ & $R$ \\
\hline$C$ & 1 & 0 & 0 & 0.634 & 0.250 & $0.400]$ \\
\hline & 0 & 1 & 0 & 0.192 & 0.250 & 0.200 \\
\hline & 0 & 0 & 1 & 0.174 & 0.500 & 0.400 \\
\hline$A$ & 0.637 & 0.582 & 0.136 & 0 & 0 & 0 \\
\hline$P$ & 0.105 & 0.109 & 0.654 & 0 & 0 & 0 \\
\hline & 0.258 & 0.309 & 0.210 & 0 & 0 & 0 \\
\hline
\end{tabular}

\begin{tabular}{|c|c|c|c|c|c|c|}
\hline & $C$ & $E$ & $M$ & $A$ & $P$ & $R$ \\
\hline$C$ & 0.5 & 0 & 0 & 0.634 & 0.250 & $0.400]$ \\
\hline $\mathbb{E}$ & 0 & 0.5 & 0 & 0.192 & 0.250 & 0.200 \\
\hline & 0 & 0 & 0.5 & 0.174 & 0.500 & 0.400 \\
\hline 4 & 0.319 & 0.291 & 0.068 & 0 & 0 & 0 \\
\hline$P$ & 0.053 & 0.055 & 0.327 & 0 & 0 & 0 \\
\hline & 0.129 & 0.155 & 0.105 & 0 & 0 & 0 \\
\hline
\end{tabular}

$\boldsymbol{C}$
$\boldsymbol{C}$
$\boldsymbol{E}$
$\boldsymbol{M}$
$\boldsymbol{A}$
$\boldsymbol{P}$
$\boldsymbol{P}$$\left[\begin{array}{cccccc}0.310 & \boldsymbol{E} & \boldsymbol{M} & \boldsymbol{A} & \boldsymbol{P} & \boldsymbol{R} \\ 0.140 & 0.140 & 0.310 & 0.310 & 0.310 & 0.310 \\ 0.216 & 0.216 & 0.216 & 0.216 & 0.216 & 0.216 \\ 0.154 & 0.154 & 0.154 & 0.154 & 0.154 & 0.154 \\ 0.095 & 0.095 & 0.095 & 0.095 & 0.095 & 0.095 \\ 0.084 & 0.084 & 0.084 & 0.084 & 0.084 & 0.084\end{array}\right]$

Since the effect of cyclicity does not exist in this example, the final priorities are directly obtained by limiting the power to converge. Although the criterion of culture also has the highest priority, the priority changes from 0.233 to 0.310 . On the other hand, the least priority is resource (0.084) instead of end-user. Compare to the priorities of the two examples, the structures play the key to both the effects and the results. In addition, it should be highlighted that when we raise the weighted matrix to limiting power, the weighted matrix should always be the stochastic matrix.

The advantage of the ANP is that it is not only appropriate for both quantitative and qualitative data types, but it also can overcome the problem of interdependence and feedback between all features. This is the reason why we adopt the ANP in our paper. However, it is clear that the key for the ANP is to determine the relationship structure between all features in advance (Lee and Kim, 2000). In next section, we discuss the procedures of ISM to construct the interdependent structures based on the relationships of the features.

\section{Interpretive structural modeling}

Interpretive structural modeling (ISM), proposed by Warfield (1974a,b, 1976) is a computer-assisted methodology to construct and understand the fundamental of the relationships of the elements in complex systems or situations. The theory of ISM is based on discrete mathematics, graph theory, social sciences, group decision-making, and computer assistance (Warfield, 1974a,b, 1976). The procedures of ISM are begun through individual or group mental models to calculate binary matrices, also called relation matrix, to present the relations of the elements.

A relation matrix can be formed by asking the question like "Does the feature $e_{i}$ inflect the feature $e_{j}$ ?" If the answer is "Yes" then $\pi_{i j}=1$, otherwise $\pi_{i j}=0$. The general form of the relation matrix can be presented as follows: 


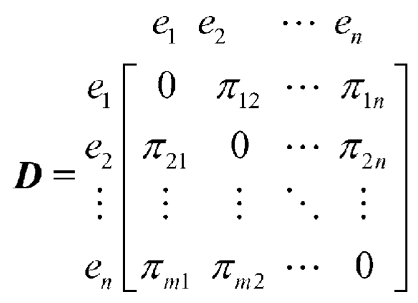

where $e_{i}$ is the $i$ th element in the system, $\pi_{i j}$ denotes the relation between $i$ th and $j$ th elements, $\boldsymbol{D}$ is the relation matrix.

After constructing the relation matrix, we can calculate the reachability matrix using Eqs. (3) and (4) as follows

$$
\boldsymbol{M}=\boldsymbol{D}+\boldsymbol{I}
$$

$$
\boldsymbol{M}^{*}=\boldsymbol{M}^{k}=\boldsymbol{M}^{k+1} \quad k>1
$$

where $\boldsymbol{I}$ is the unit matrix, $k$ denotes the powers, and $\boldsymbol{M}^{*}$ is the reachability matrix. Note that the reachability matrix is under the operators of the Boolean multiplication and addition (i.e. $1 \times 1=1$ and $1+1=1$ ). For example

$$
M=\left(\begin{array}{ll}
1 & 0 \\
1 & 1
\end{array}\right), \quad M^{2}=\left(\begin{array}{ll}
1 & 0 \\
1 & 1
\end{array}\right)
$$

Next we can calculate the reachability set and the priority set based on Eqs. (5) and (6), respectively, as the following equations

$R\left(t_{i}\right)=\left\{e_{i} \mid m_{j i}^{*}=1\right\}$

$A\left(t_{i}\right)=\left\{e_{i} \mid m_{i j}^{*}=1\right\}$

where $m_{i j}$ denotes the value of the $i$ th row and the $j$ th column.

Then, according to Eq. (7), the levels and relationships between the elements can be determined and the structure of the elements' relationships can also be expressed using the graph.

$R\left(t_{i}\right) \cap A\left(t_{i}\right)=R\left(t_{i}\right)$

Next, we also use a simple example to show the steps of ISM for more understanding. Assume the ecosystem consist of water $(W)$, fish $(F)$, hydrophytes $(H)$, and fisherman $(M)$ and the relationships can be expressed as the following graph and relation matrix (Fig. 5).

Then, the relation matrix adds the identity matrix to form the $\boldsymbol{M}$ matrix as follows:

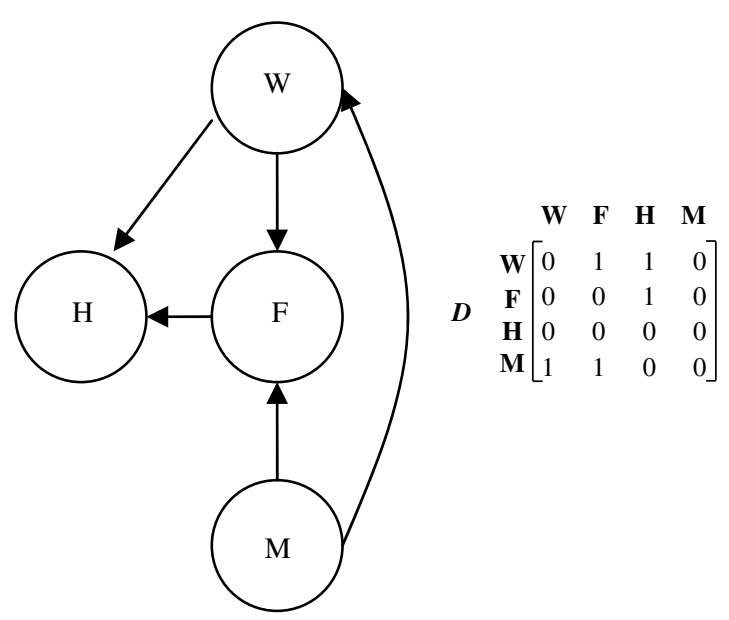

Fig. 5. The relationships of the elements.

$$
\boldsymbol{M}=\boldsymbol{D}+\boldsymbol{I}=\left[\begin{array}{llll}
1 & 1 & 1 & 0 \\
0 & 1 & 1 & 0 \\
0 & 0 & 1 & 0 \\
1 & 1 & 0 & 1
\end{array}\right]
$$

Last, the reachability matrix are obtained by powering the matrix, $\boldsymbol{M}$, to satisfy the Eq. (4).

$$
\boldsymbol{M}^{*}=\boldsymbol{M}^{2}=\boldsymbol{M}^{2+n}=\left[\begin{array}{llll}
1 & 1 & 1 & 0 \\
0 & 1 & 1 & 0 \\
0 & 0 & 1 & 0 \\
1 & 1 & 1^{*} & 1
\end{array}\right], \quad n=1,2, \ldots
$$

where the $\operatorname{star}(*)$ indicates the derivative relation which does not emerge in the original relation matrix. In order to determine the levels of the elements in a hierarchical structure, the reachability set and the priority set are derived based on Eqs. (4) and (5).

Then, the first level can be derived according to Eq. (7) and is the fisherman. The other levels can also be determined with the same procedures (Table 1).

The final results of the relationships of the elements based on rechability matrix and Table 2 can be plotted as the following graph (Fig. 6).

Note that the relationships between the fisherman and the hydrophytes are generated by the reachability matrix. In addition, since hierarchical structural analysis (HSA) and fuzzy ISM (FISM) 
Table 1

The reachability set and the priority set

\begin{tabular}{llll}
\hline$e_{i}$ & $R\left(t_{i}\right)$ & $A\left(t_{i}\right)$ & $R\left(t_{i}\right) \cap A\left(t_{i}\right)$ \\
\hline 1 & 1,4 & $1,2,3$ & 1 \\
2 & $1,2,4$ & 2,3 & 2 \\
3 & $1,2,3,4$ & 4 & 4 \\
4 & 4 & $1,2,3,4$ & 4 \\
\hline
\end{tabular}

Table 2

Levels in the ecosystem

\begin{tabular}{ll}
\hline Level 1 & Fisherman \\
Level 2 & Water \\
Level 3 & Fish \\
Level 4 & Hydrophytes \\
\hline
\end{tabular}

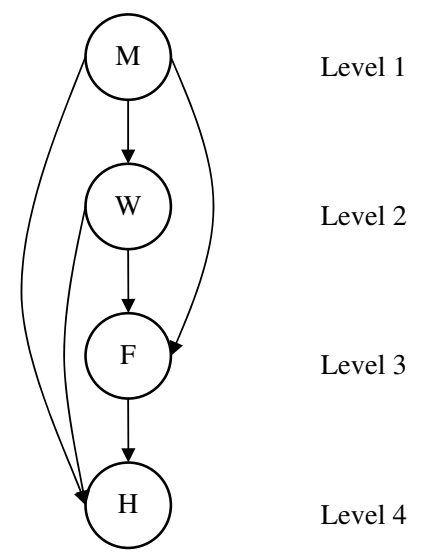

Fig. 6. Hierarchical structure of the elements.

(Ohuchi and Kaji, 1989; Wakabayashi et al., 1995; Ohuchi et al., 1988) have been proposed to extend ISM to the feedback structure and the fuzzy environment, we can determine the various network structures in practice.

For completeness, we discuss the whole procedures of ISM. Since we focus on the network structure rather than the hierarchical structure in this paper, the procedures of determining the levels are ignored. So we can just plot the network structure based on the rechability matrix and ignore the results of the reachability set and the priority set. Using the relation matrix and the reachability matrix, we can easily construct the relationships of the elements. Then, on the basis of the relation- ships, we can obtain the weights using the ANP. In next section, a numerical example is used to illustrate the processes of the proposed method.

\section{Numerical example}

Here, we provide a numerical example to demonstrate the proposed method. In order to obtain our results, the first step is to construct the network structure using the ISM procedures. The second step is to calculate the limited matrix and to obtain the feature's weights based on the network structure in the ANP. The last step is to obtain the final weighted proximity matrix according to the feature's weights, and then to proceed with MDS analysis.

This numerical example examines the closeness of six products using MDS analysis. As we know, the closeness of the products is the very important information for firms. On the basis of the results, decision-makers can identify the directly competitive products and the product position in the market. In this example, we assume that there are five features to be evaluated, including the price, the package, the location, the function, and the manufacturer for measuring the closeness of the products. The proximity matrices of the five features are shown in Appendix A.

In order to compare with the conventional MDS, we first assume the weights of all features are equal. Then, we can calculate the proximity matrix based on Tables A.1-A.5 and the results can be shown as in Table 3 .

On the basis of the proximity matrix, we can use the conventional MDS procedures to obtain the coordinates of two-dimensional configurations of the products as shown in Table 4.

Table 3

The conventional proximity matrix

\begin{tabular}{lllllll}
\hline$\Delta^{*}$ & $\mathrm{~A}$ & $\mathrm{~B}$ & $\mathrm{C}$ & $\mathrm{D}$ & $\mathrm{E}$ & $\mathrm{F}$ \\
\hline $\mathrm{A}$ & 0 & 4.6 & 4.4 & 4 & 5.8 & 5.6 \\
$\mathrm{~B}$ & 4.6 & 0 & 2.6 & 5.8 & 5.2 & 3.2 \\
$\mathrm{C}$ & 4.4 & 2.6 & 0 & 4.8 & 3.8 & 4 \\
$\mathrm{D}$ & 4 & 5.8 & 4.8 & 0 & 4.8 & 7 \\
$\mathrm{E}$ & 5.8 & 5.2 & 3.8 & 4.8 & 0 & 3 \\
$\mathrm{~F}$ & 5.6 & 3.2 & 4 & 7 & 3 & 0 \\
\hline
\end{tabular}


Table 4

Coordinates of two-dimensional configurations

\begin{tabular}{lcc}
\hline Object & Dimension 1 & Dimension 2 \\
\hline A & 0.02 & 0.83 \\
B & 1.58 & -0.50 \\
C & -1.75 & 0.73 \\
D & -0.10 & -0.95 \\
E & 1.06 & 1.02 \\
F & -0.80 & -1.12 \\
\hline
\end{tabular}

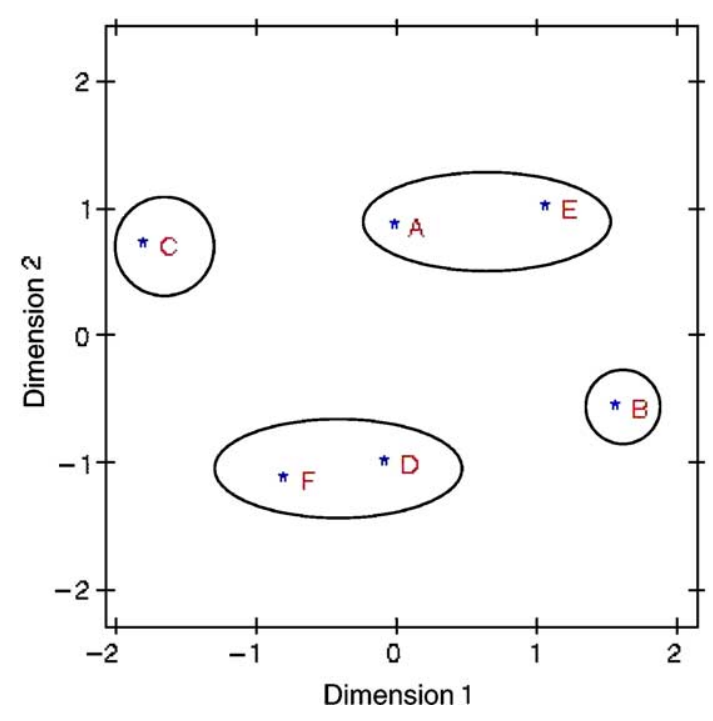

Fig. 7. Two-dimensional relationship mapping.
Next, we use the two-dimensional mapping to visually show the relationships of the products as the following figure.

On the basis of Fig. 7, we can conclude that there are four segments in this market using the conventional MDS analysis. The product A and the product $\mathrm{E}$ belong to the same cluster (i.e. the direct competitor) and the product $\mathrm{F}$ and the product $\mathrm{D}$ belong to the same cluster. The product $\mathrm{B}$ or the product $\mathrm{C}$ belongs to the niche market.

Next, we use the proposed method to consider the effects of the weights in the MDS analysis. First, we will judge the relationships of the features, which can be done by our knowledge or the expert's opinions to form the relation matrix, $\boldsymbol{D}$, as shown in Table 5 .

From the relation matrix, we can calculate the reachability matrix, $\boldsymbol{M}^{*}$, based on Eqs. (3) and (4) and as shown in Table 6. The reachability matrix presents the relationships of all features, and we can construct the network structure based on Table 6.

Based on Table 6, the network relationships can be constructed as shown in Fig. 8.

Now, based on the structure as shown in Fig. 8, we can process the pairwise comparisons between the features (Appendix B) to form the following supermatrix.

Table 5

The relation matrix between features

\begin{tabular}{llllll}
\hline $\boldsymbol{D}$ & Price & Package & Location & Function & Manufacturer \\
\hline Price & 0 & 1 & 0 & 1 & 0 \\
Package & 1 & 0 & 0 & 0 & 0 \\
Location & 1 & 0 & 0 & 0 & 0 \\
Function & 1 & 0 & 0 & 0 & 0 \\
Manufacturer & 1 & 0 & 0 & 0 & 0 \\
\hline
\end{tabular}

Table 6

The reachability matrix between features

\begin{tabular}{llllll}
\hline $\boldsymbol{M}^{*}$ & Price & Package & Location & Function & Manufacturer \\
\hline Price & 1 & 1 & 0 & 1 & 0 \\
Package & 1 & 1 & 0 & $1 *$ & 0 \\
Location & 1 & $1^{*}$ & 1 & $1 *$ & 0 \\
Function & 1 & $1^{*}$ & 0 & 1 & 0 \\
Manufacturer & 1 & $1^{*}$ & 0 & $1^{*}$ & 1 \\
\hline
\end{tabular}

*Indicates the relationship produced by the reachability matrix. 


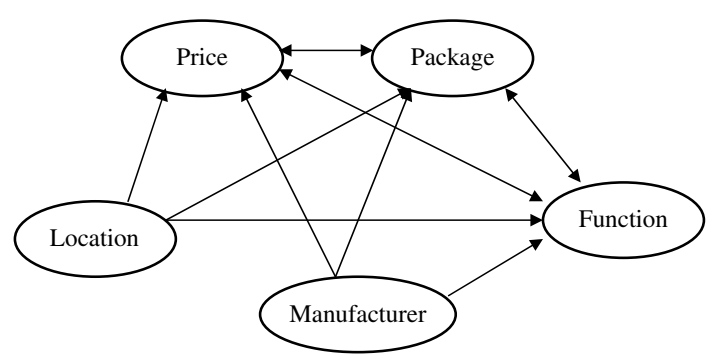

Fig. 8. Network structure.

Function Location Manufacturer Package Price

Function
Location
Manufacturer
Package
Price $\left[\begin{array}{ccccc}0 & 0.185 & 0.451 & 0.250 & 0.875 \\ 0 & 0 & 0 & 0 & 0 \\ 0 & 0 & 0 & 0 & 0 \\ 0.167 & 0.156 & 0.059 & 0 & 0.125 \\ 0.833 & 0.659 & 0.490 & 0.75 & 0\end{array}\right]$

Then, the supermatrix is raised to limiting powers to obtain the weights of all features. The weight of each feature can be shown in Table 7 based on the results of the limited matrix.

Using Eq. (8), we can obtain the weighted proximity matrix to consider the network relationships of the features.

$\Delta_{i j}^{*}=\sum_{k=1}^{m} w_{k} \Delta_{i j k}, \quad \Delta^{*}=\left[\Delta_{i j}^{*}\right]$

where $\Delta^{*}$ denotes the weighted proximity matrix, $w_{k}$ denotes the feature's weight, and $\Delta_{i j}$ denotes the proximity matrix according to the $i$ th feature and the $j$ th object. The weighted proximity matrix of the six products is represented as shown in Table 8 .

Finally, with the same procedures as like as in the conventional MDS analysis, the two-dimensional configurations and the two-dimensional relationship mapping can be obtained as shown in Table 9 and Fig. 9, respectively.

Table 7

The weights of the features using the ANP

\begin{tabular}{llllll}
\hline & Price & Package & Location & Function & Manufacturer \\
\hline $\boldsymbol{w}^{\mathrm{T}}$ & 0.424 & 0.000 & 0.000 & 0.127 & 0.449 \\
\hline
\end{tabular}

Table 8

The weighted proximity matrix

\begin{tabular}{lllllll}
\hline$\Delta^{*}$ & $\mathrm{~A}$ & $\mathrm{~B}$ & $\mathrm{C}$ & $\mathrm{D}$ & $\mathrm{E}$ & $\mathrm{F}$ \\
\hline $\mathrm{A}$ & 0 & 2.771 & 4.821 & 3.254 & 5.467 & 6.415 \\
$\mathrm{~B}$ & 2.771 & 0 & 2.322 & 5.17 & 5.179 & 4.075 \\
$\mathrm{C}$ & 4.821 & 2.322 & 0 & 5.204 & 4.424 & 2.737 \\
$\mathrm{D}$ & 3.254 & 5.17 & 5.204 & 0 & 5.696 & 6.424 \\
$\mathrm{E}$ & 5.467 & 5.179 & 4.424 & 5.696 & 0 & 2.728 \\
$\mathrm{~F}$ & 6.415 & 4.075 & 2.737 & 6.424 & 2.728 & 0 \\
\hline
\end{tabular}

As shown in Fig. 7, there are only three segments in this market. The product $\{A, E\}$, $\{B, D\}$, or $\{F, C\}$ has the similar position and belong to the same cluster. Compare with the results of the conventional MDS analysis, we can conclude that the results show the significant differences when considering the effects of the weights. In next section, we provide the detailed discussions and comparisons between the conventional MDS and the proposed method.

\section{Discussions and comparisons}

MDS analysis is a wildly useful tool to descript the relative locations between objectives.

Table 9

Coordinates of two-dimensional configurations using the ANP

\begin{tabular}{lcc}
\hline Object & Dimension 1 & Dimension 2 \\
\hline A & 1.62 & 0.47 \\
B & -1.08 & -0.94 \\
C & -0.54 & 1.33 \\
D & 1.46 & -0.44 \\
E & -1.21 & 0.53 \\
F & -0.25 & -0.94 \\
\hline
\end{tabular}




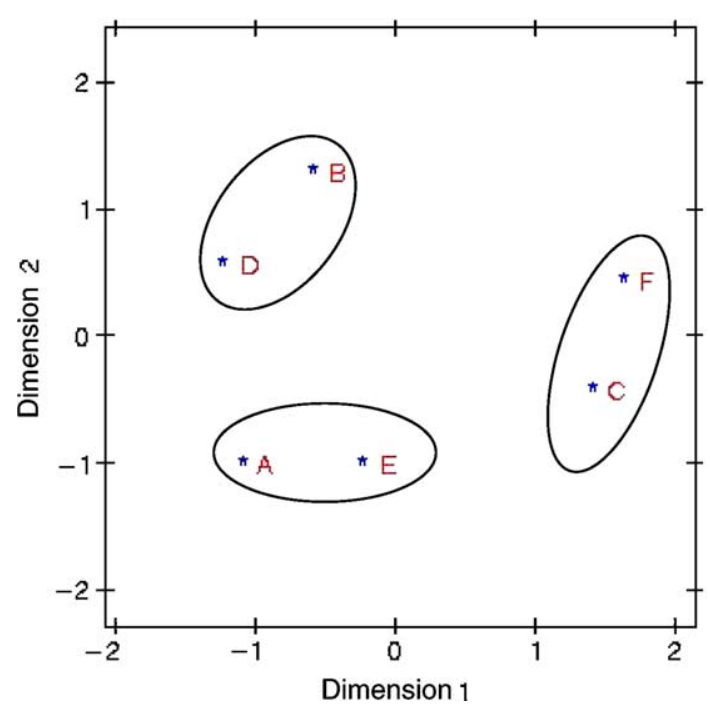

Fig. 9. Two-dimensional relationship mapping using the ANP.

In the conventional MDS, the weights of the features usually are ignored. However, it may be a big problem when the feature has the interdependent relationships. In our numerical example, it is clear that the results of the conventional MDS and the proposed method have the significant differences.

The problem of our example is to identify the similar degrees of the six products using the MDS procedures. The proximate scores are given based on the five features (the price, the package, the location, the function, and the manufacturer). In the conventional MDS, all features are treated with the same weights. However, it is clear that the package and the location features do not affect the similar degree of the products. Using the ANP method, the weights are more close to the real world situation. The significant differences can also be found in our numerical example.

Another advantage of the proposed method is that we can understand the relationships between all features according to the results of the ISM procedures. Understanding the relationship structure among features is also important for decision makers. Therefore, the proposed method can provide the more informative and accurate results.

\section{Conclusions}

Although MDS is a useful statistical tool to understand the relationships between the objects visually, it is difficult for humans to judge the proximity matrix in a complex system or situation. Even through we can divide a complex system into subsystems, in practice, the features or criteria in these subsystems usually exist interdependence or feedback, so it is hard to deal with the conventional statistical tools.

The ANP is used to overcome these problems, and has been widely used in various fields. By calculating the supermatrix, we can obtain the global priority vectors. However, the results are various with the different network structures. In this paper, the ISM procedures are used to overcome the problem by calculating the relation matrix and reachability matrix to construct the network relationships between the features. Then, the weighted matrix is obtained using the ANP analysis based on the results of ISM. Finally, the weighted proximity matrix is obtained by the ANP and is used for the MDS analysis. On the basis of the numerical results, we can conclude that the proposed method can provide the more informative and accurate results than the conventional MDS analysis.

\section{Appendix A}

The proximity matrices of price, package, location, function and manufacturer are given using 9 scaling by expert's opinions and are presented in Tables A.1-A.5.

Table A.1

The proximity matrix by the price feature

\begin{tabular}{lllllll}
\hline Price & A & B & C & D & E & F \\
\hline A & 0 & 2 & 2 & 3 & 7 & 5 \\
B & 2 & 0 & 2 & 6 & 8 & 1 \\
C & 2 & 2 & 0 & 7 & 5 & 3 \\
D & 3 & 6 & 7 & 0 & 8 & 7 \\
E & 7 & 8 & 5 & 8 & 0 & 1 \\
F & 5 & 1 & 3 & 7 & 1 & 0 \\
\hline
\end{tabular}


Table A.2

The proximity matrix by the package feature

\begin{tabular}{lllllll}
\hline Package & A & B & C & D & E & F \\
\hline A & 0 & 8 & 3 & 5 & 9 & 7 \\
B & 8 & 0 & 5 & 6 & 1 & 1 \\
C & 3 & 5 & 0 & 3 & 2 & 7 \\
D & 5 & 6 & 3 & 0 & 4 & 9 \\
E & 9 & 1 & 2 & 4 & 0 & 1 \\
F & 7 & 1 & 7 & 9 & 1 & 0 \\
\hline
\end{tabular}

Table A.3

The proximity matrix by the location feature

\begin{tabular}{lllllll}
\hline Location & $\mathrm{A}$ & $\mathrm{B}$ & $\mathrm{C}$ & $\mathrm{D}$ & $\mathrm{E}$ & $\mathrm{F}$ \\
\hline $\mathrm{A}$ & 0 & 8 & 6 & 4 & 6 & 5 \\
$\mathrm{~B}$ & 8 & 0 & 2 & 9 & 8 & 3 \\
C & 6 & 2 & 0 & 4 & 4 & 1 \\
D & 4 & 9 & 4 & 0 & 4 & 7 \\
E & 6 & 8 & 4 & 4 & 0 & 5 \\
F & 5 & 3 & 1 & 7 & 5 & 0 \\
\hline
\end{tabular}

Table A.4

The proximity matrix by the function feature

\begin{tabular}{lllllll}
\hline Function & A & B & C & D & E & F \\
\hline A & 0 & 1 & 3 & 5 & 2 & 2 \\
B & & 0 & 1 & 3 & 7 & 4 \\
C & & & 0 & 7 & 4 & 8 \\
D & & & & 0 & 4 & 6 \\
E & & & & & 0 & 4 \\
F & & & & & & 0 \\
\hline
\end{tabular}

Table A.5

The proximity matrix by the manufacturer feature

\begin{tabular}{lllllll}
\hline Manufacturer & A & B & C & D & E & F \\
\hline A & 0 & 4 & 8 & 3 & 5 & 9 \\
B & & 0 & 3 & 5 & 2 & 7 \\
C & & & 0 & 3 & 4 & 1 \\
D & & & & 0 & 4 & 6 \\
E & & & & & 0 & 4 \\
F & & & & & & 0 \\
\hline
\end{tabular}

\section{Appendix B}

The comparison of the features can be described as in Table B.1.
Table B.1

The comparison of the features

\begin{tabular}{llll}
\hline Criterion & $X$ & $\begin{array}{l}\text { For criterion, } \\
\text { how much } X \text { is more } \\
\text { important than } Y \text { ? }\end{array}$ & $Y$ \\
\hline Price & Function & 7 & Package \\
Location & Function & 1 & Package \\
Location & Function & $1 / 3$ & Price \\
Location & Package & $1 / 5$ & Price \\
Manufacturer & Function & 7 & Package \\
Manufacturer & Function & 1 & Price \\
Manufacturer & Package & $1 / 9$ & Price \\
Function & Package & $1 / 5$ & Price \\
Package & Function & $1 / 3$ & Price \\
\hline
\end{tabular}

\section{References}

Arabic, P., Carroll, J.D., DeSarbo, W.S., 1987. Three-Way Scaling and Clustering, Sage University Paper Series on Quantitative Application in the Social Sciences. Sage Publications, Beverly Hills and London.

Karsak, E.E., Sozer, S., Alptekin, S.E., 2002. Product planning in quality function deployment using a combined analytic network process and goal programming approach. Comput. Ind. Eng. 44 (1), 171-190.

Kruskal, J.B., Wish, M., 1978. Multidimensional Scaling, Sage University Paper Series on Quantitative Application in the Social Sciences. Sage Publications, Beverly Hills and London.

Lee, J.W., Kim, S.H., 2000. Using analytic network process and goal programming for interdependent information system project selection. Comput. Oper. Res. 27 (4), 367-382.

Mead, A., 1992. Review of the development of multidimensional scaling methods. Statistician 41 (1), 27-39.

Meade, L.M., Presley, A., 2002. R\&D project selection using the analytic network process. IEEE Trans. Eng. Manage. 49 (1), 59-66.

Momoh, J.A., Zhu, J., 2003. Optimal generation scheduling based on AHP/ANP. IEEE Trans. Systems Man Cybernet.-Part B: Cybernet. 33 (3), 531-535.

Ohuchi, A., Kaji, I., 1989. Correction procedures for flexible interpretive structural modeling. IEEE Trans. Systems Man Cybernet. 19 (1), 85-94.

Ohuchi, A., Kase, S., Kaji, I., 1988. MINDS: A flexible interpretive structural modeling system. In: Proc. 1988 IEEE Internat. Conf. on Systems, Man, and Cybernetics, vol. 2, pp. 1326-1329.

Saaty, T.L., 1980. The Analytic Hierarchy Process. McGrawHill, New York.

Saaty, T.L., 1996. Decision Making with Dependence and Feedback: The Analytic Network Process. RWS Publications, Pittsburgh.

Saaty, T.L., Vargas, L.G., 1998. Diagnosis with dependent symptoms: Bayes theorem and the analytic hierarchy process. Oper. Res. 46 (4), 491-502. 
Sarkis, J., 2003. A strategic decision framework for Green supply chain management. J. Cleaner Production 11 (4), 397-409.

Schiffman, S.S., Reynolds, M.L., Young, F.W., 1981. Introduction to Multidimensional Scaling. Academic Press, New York.

Sekitani, K., Takahashi, I., 2001. A unified model and analysis for AHP and ANP. J. Oper. Res. Soc. Jpn. 44 (1), 67-89.

Wakabayashi, T., Itoh, L., Ohuchi, A., 1995. A method for constructing system models by fuzzy flexible interpretive structural modeling fuzzy systems. In: Internat. Joint Conf. of the 4th IEEE Internat. Conf. on Fuzzy Systems and The
Second Internat. Fuzzy Engineering Symposium, Proc. 1995 IEEE Internat. Conf. on, Vol. 2, pp. 913-918.

Warfield, J.N., 1974a. Toward interpretation of complex structural modeling. IEEE Trans. Systems Man Cybernet. 4 (5), 405-417.

Warfield, J.N., 1974b. Developing Interconnection Matrices in Structural Modeling. IEEE Trans. Systems Man Cybernet. 4 (1), 81-87.

Warfield, J.N., 1976. Societal Systems: Planning, Policy, and Complexity. Wiley Interscience, New York.

Young, F.W., Hamer, R.M., 1987. Multidimensional Scaling: History, Theory, and Applications. Lawrence Erlbaum Associates, Hillsdale, NJ. 